\title{
A comprehensive study of a versatile magnetic refrigeration demonstrator
}

\author{
D. Velázquez, C. Estepa, E. Palacios, R. Burriel \\ Instituto de Ciencia de Materiales de Aragón (ICMA), CSIC - Universidad de Zaragoza, Pedro Cerbuna 12, 50009 Zaragoza, Spain, +34 976 \\ 762467, +34976 761229, davelazq@unizar.es
}

\begin{abstract}
A versatile room temperature reciprocating magnetic refrigeration demonstrator has been designed, built and tested in order to evaluate the influence of different running parameters and to check suitable magnetocaloric materials for cooling at room temperature. A comprehensive study has been done with $\mathrm{Gd}$ spheres of 0.2-0.4 mm diameter arranged as a double regenerator with 15 g each. A Halbach $\mathrm{Nd}_{2} \mathrm{Fe}_{14} \mathrm{~B}$ permanent magnet with a slot of $10 \mathrm{~mm}$ width has been used to generate the magnetic field with a maximum value of $1.4 \mathrm{~T}$. The heat transfer fluid is a mixture of water and ethylene glycol in a 75-25 percentage. The demonstrator achieves a maximum no-load temperature span close to $20 \mathrm{~K}$, with a regeneration ratio of $\xi=4.1$, and a maximum cooling power $\dot{Q}_{c}=6 \mathrm{~W}$ at zero temperature span. $C O P$ values have been shown and different thermodynamic AMR cycles have been studied looking for the best parameters.
\end{abstract}

Keywords: Magnetic refrigerator, Prototype, Experimentation, Thermodynamic cycle, Gadolinium

\section{Introduction}

Magnetic refrigeration is a novel technology which is trying to be an alternative to conventional gas compression - expansion cooling systems at room temperature. This technology is more environmental friendly because it does not use CFC gases, which are partially responsible for the ozone layer depletion and the enhancement of the greenhouse effect. In addition, it has the potential to lower the energy consumption by $20-30 \%$, as reported by Gschneidner and Pecharsky [1].

Many prototypes have already been built and tested successfully. Most of them are composed of plates or spheres of gadolinium as an active refrigerant because is a well known benchmark material. They have been widely reported by $\mathrm{Yu}$ et al. [2], and Gschneidner and Pecharsky [1]. Some of them are test devices to check magnetocaloric materials and operation parameters, and others are closer to commercial machines to achieve useful temperature spans and cooling powers for applications at room temperature. According to the simple classification given by Gschneidner and Pecharsky [1], test devices are from the type of the first generation systems and were the first to be built. The magnetisation - demagnetisation steps are reciprocating and versatile in order to test and optimise the operation parameters. The main limitation is the frequency because of the reciprocating motion, so, low cooling power is achieved. Second and third generation systems are rotary and achieve higher frequencies, thus higher cooling power. Second generation systems rotate the magnetic beds and third generation rotate the permanent magnet assembly. More elaborated classifications of systems have been reported, as seen in a recent publication by Scarpa et al. [3], that take into account many different parameters of the refrigeration device.

Several results have been presented from test devices, showing varying success. Some of the systems show only a proof of concept of magnetic refrigeration without optimising parameters or doing any further study, as 


\begin{tabular}{|c|c|c|c|}
\hline \multirow{2}{*}{\multicolumn{2}{|c|}{ Nomenclature }} & $t$ & time $(s)$ \\
\hline & & $U$ & utilization factor (-) \\
\hline \multicolumn{2}{|c|}{ Standard } & $V_{d}$ & dead volume of the regenerator $\left(\mathrm{cm}^{3}\right)$ \\
\hline$C O P$ & coefficient of performance (-) & $V_{f}$ & pushed fluid volume $\left(\mathrm{cm}^{3}\right)$ \\
\hline$c_{f}$ & fluid specific heat $\left(\mathrm{J} \mathrm{kg}^{-1} \mathrm{~K}^{-1}\right)$ & $V_{i}$ & interstitial volume of the regenerator $\left(\mathrm{cm}^{3}\right)$ \\
\hline & solid specific heat $\left(\mathrm{J} \mathrm{kg}^{-1} \mathrm{~K}^{-1}\right)$ & $\xi$ & regeneration ratio (-) \\
\hline$\Delta T$ & temperature span of the regenerator $(\mathrm{K})$ & & \\
\hline$\Delta T_{M C E}$ & adiabatic temperature change $(\mathrm{K})$ & \multicolumn{2}{|c|}{ Abbreviations } \\
\hline$f$ & frequency $(\mathrm{Hz})$ & AMR & active magnetic regenerator \\
\hline$g$ & gravitational acceleration $\left(\mathrm{m} \mathrm{s}^{-2}\right)$ & $\mathrm{CB}$ & cold blow \\
\hline$H_{0}$ & zero magnetic field $(\mathrm{T})$ & $\mathrm{D}$ & demagnetisation process \\
\hline$H_{\max }$ & maximum magnetic field (T) & $\mathrm{HB}$ & hot blow \\
\hline$L$ & length of the regenerator $(\mathrm{mm})$ & M & magnetisation process \\
\hline$m_{f}$ & fluid mass (kg) & $\mathrm{PF}$ & programming the positions of the pistons \\
\hline$m_{s}$ & solid mass $(\mathrm{kg})$ & PM & programming the position of the magnet \\
\hline$\dot{m}$ & mass flow rate $\left(\mathrm{g} \mathrm{s}^{-1}\right)$ & & \\
\hline$\dot{Q}_{c}$ & cooling power $(\mathrm{W})$ & \multicolumn{2}{|l|}{ Indices } \\
\hline$R_{v d}$ & dead volume ratio (-) & $\mathrm{f}$ & final \\
\hline$R_{v f}$ & pushed fluid volume ratio (-) & $\mathrm{i}$ & initial \\
\hline
\end{tabular}

presented by Lu et al. [4], Bour et al. [5], and Chiba et al. [6]. Other publications as Plaznik et al. [7] and Tušek et al. [8], not only show successful results, but also a comprehensive experimental and simulation study regarding the influence of different thermodynamic cycles and geometries of the active magnetic regenerator. In general, a more advanced study is required for a deeper understanding of the technology.

In this paper a versatile reciprocating magnetic refrigeration machine to test active refrigerants is presented. A comprehensive study of the parameters has been done with gadolinium as the active material, optimising the temperature span and the cooling capacity of the system. Also the $C O P$ has been measured, although this optimisation has not been the aim of this system. Finally, several thermodynamic cycles have been tested and compared.

\section{Active magnetic regenerator design}

There are many parameters to be taken into account in the design of a magnetic refrigerator system. The type of system should be chosen according to the required performance and the aim of the project. The performance is mainly defined by the temperature span reached between cold and hot side, the cooling power and the coefficient of performance. The $C O P$ is an industry standard, defined as the ratio between the cooling power and the total power consumption to achieve it. A reciprocating system should be suitable for a comprehensive study of the operation parameters, whereas a rotary system, that allows reaching higher frequencies and then higher cooling powers, should be suitable to fulfil some of the performance requirements of a specific application or even all of them.

An efficient design of the permanent magnet by simulations is needed. Bjørk et al. [9] defined a performance parameter to optimise it. The magnetic field in the high field region, the available volume to place magnetocaloric material and the fraction of the cycle in which the magnet is being used have to be maximised, while the magnetic field in the low field region and the magnet volume have to be minimised. Moreover, the work related to the motion for applying the high and zero magnetic field should be included in the simulations and minimised considering also the $C O P$.

The active magnetic regenerator should also be simulated. The optimisation of its geometry and porosity allows to maximise the solid - liquid heat transfer and therefore the performance of the system. As a result of the simulation, the range of the operation parameters such as frequency and pushed fluid volume, are defined. In general, effective heat transfer configurations lead to higher pressure drop through the regenerator, and therefore higher input work, so a compromise should be achieved if the $C O P$ is considered.

Finally, there are a few technical issues related to the regenerator to be followed. These define the range of 
a)

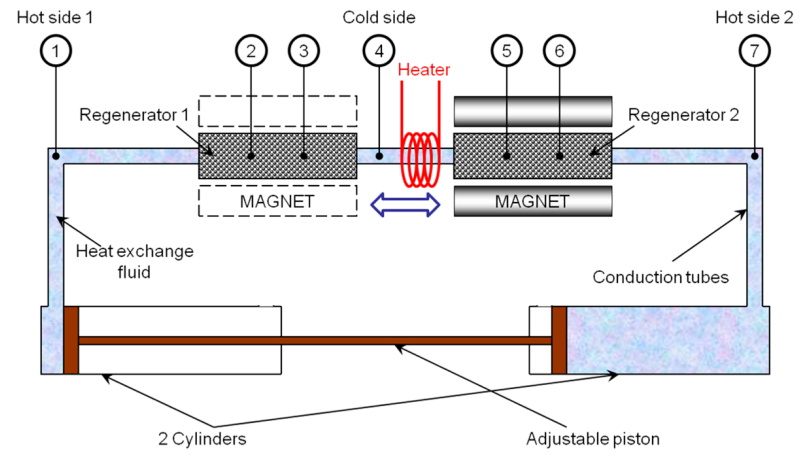

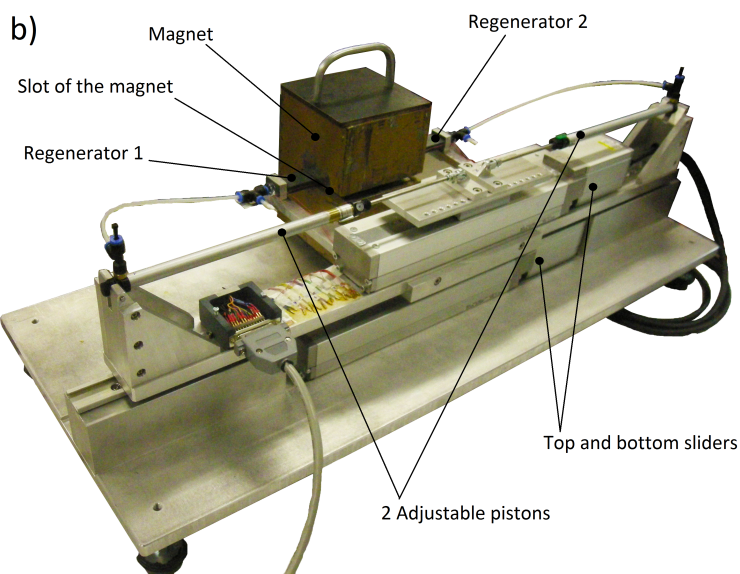

Figure 1: Scheme of the magnetic refrigeration demonstrator (a) and picture of the complete system (b)

the operation parameters for a given regenerator.

The utilization factor is an important parameter, defined in Eq. 1, and represents the ratio between the thermal capacities of the pushed liquid and the active material. It is critical to design the regenerator in order to obtain values around 1 , which means that the system is thermodynamically compensated,

$$
U=\frac{m_{f} c_{f}}{m_{s} c_{s}}
$$

where $m_{f}$ is the pushed fluid mass, $c_{f}$ is the fluid specific heat, $m_{s}$ is the mass of magnetocaloric material and $c_{s}$ is its zero field specific heat at the Curie temperature.

Also, the fluid system has to be designed to ensure the smallest possible dead volume ratio. The larger this parameter, which is defined in Eq. 2, the worse the system performance.

$$
R_{V d}=\frac{V_{d}}{V_{i}}
$$

where $V_{i}$ is the interstitial volume of the regenerator (volume filled by refrigerant liquid) and $V_{d}$ is the dead volume. $V_{d}$ represents, in the case of bidirectional fluid movement to the sinks, the volume contained between the hot or the cold side and the regenerator. However, if the fluid movement is unidirectional, $V_{d}$ is the volume between the regenerator and the derivation of the unidirectional flow. A system with unidirectional flow outside of the regenerator based on check valves has been shown by Tura and Rowe [10]. The dead volume prevents some pushed fluid from the regenerator to reach the cold or hot side, wasting some effect of the active magnetic regenerator, therefore an efficient design should minimise it.
Last, the pumping system has to be able to adjust the pushed fluid volume ratio, defined in Eq. 3, between limits allowing an efficient heat transfer to the cold and hot sides and maintaining the required temperature span, as explained below.

$$
R_{V f}=\frac{V_{f}}{V_{i}}
$$

where $V_{f}$ is the pushed fluid volume in each blow. It is obvious that $R_{V f}$ should be higher than $R_{V d}$, otherwise none of the fluid from the regenerator reaches the cold and hot sides and no significant temperature spans will be achieved between them. Besides, in order to obtain wide temperature spans in systems using fluid with a high thermal capacity compared to the solid one, $R_{V f}$ should be lower than $1+R_{V d}$ to avoid mixing fluid from the cold and hot sides. If the aim is to achieve high cooling power, it could be desirable to make $R_{V f}>1+$ $R_{V d}$, because the cooling power is strongly dependant on the pushed fluid mass and can increase with $R_{V f}$ until the regenerator capacity is overwhelmed. But it should be noticed that the temperature span will be reduced for these high ratios.

A good regenerator design and selection of its technical parameters allow to maximise its performance. A parameter to measure the effectiveness of the active magnetic regenerator, regardless of the magnetocaloric material is the regeneration ratio, which is defined in Eq. 4 , as follows.

$$
\xi=\frac{\Delta T}{\Delta T_{M C E}}
$$

where $\Delta T$ is the regenerator temperature span achieved between the hot and the cold sides in the steady state and 
$\Delta T_{M C E}$ is the adiabatic temperature change at the Curie temperature upon the application of magnetic field.

\section{Demonstrator}

A versatile magnetic demonstrator has been built in order to check the magnetic refrigeration potential. Several parameters can be modified in a broad range as the pushed fluid volume $\left(V_{f}\right)$, mass flow rate $(\dot{m})$ and heat load $\left(\dot{Q}_{c}\right)$. It is a first generation magnetic refrigeration system characterised by a reciprocating magnetisation and demagnetisation motion. A sketch of the system is shown in Fig. 1(a). It is composed of a double active magnetic regenerator looking for an improved efficiency, as can be seen in Richard et al. [11], where a regenerator is introduced inside the magnetic field (magnetisation) as the other one is removed from it (demagnetisation) simultaneously, using the magnetic field all the time. The pumping system is composed of two adjustable pistons to push the refrigerant liquid through the regenerators. The cold side is placed in between both regenerators, where there is an electrical coil heater simulating the cooling power. Then, there are two hot sides on the other end of the regenerators that include the volume of the piston and the connecting tubes. The resulting volumes for the cold and hot side are 0.42 and $4.11 \mathrm{~cm}^{3}$, respectively in the configuration used for our experiments. A complete temperature measurement system is installed in order to record the temperature in the hot and cold sides, and inside the regenerators at $1 / 3$ and $2 / 3$ of their lengths. The hot side probes are located to some distance from the regenerators because their bonded parts are not accessible due to the reduced slot of the magnet. So, the tube volume from the probe to the regenerator is considered as the dead volume of the hot side. A picture of the system can be seen in the Fig. 1(b), with all the parts of the demonstrator discussed above.

The refrigeration system performs an active magnetic regenerator (AMR) cycle, which is based on overlapping Brayton cycles for the solid elements contained in the regenerator, as is explained in Tishin and Spichkin [12]. The cycle is composed of two adiabatic and two isofield steps. As it is a double active magnetic regenerator system, two equivalent cycles are done at the same time by the regenerators but with a phase shift of half a cycle, so, just one regenerator thermodynamic cycle is described below. Firstly, the regenerator is adiabatically magnetised, which produces a temperature rise of the magnetocaloric material. Secondly, there is a hot blow, where the liquid is pushed through the regenerator to the hot side. The regenerator transfers heat to the liquid, which carries some heat to the hot side. After that, the regenerator is adiabatically demagnetised with a consequent decrease of its temperature. Finally a cold blow is done, where the liquid is pushed through the regenerator to the cold side. The regenerator absorbs heat from the liquid, which removes some heat from the cold side. Frequency values are referred to this complete cycle, but it has to be noted that two cycles are done at the same time, so, the cooling power values can be doubled because it works like a double refrigeration system.

\subsection{Magnet}

The magnetic field source is a Halbach permanent magnet of $\mathrm{Nd}_{2} \mathrm{Fe}_{14} \mathrm{~B}(120 \times 127 \times 176) \mathrm{mm}^{3}$ with a slot of $10 \mathrm{~mm}$ high and $(120 \times 79) \mathrm{mm}^{2}$ of surface. It weights around $20 \mathrm{~kg}$ and remains still while the regenerators move in and out of the magnet to be magnetised and demagnetised. This translation is performed by a bottom slider which displaces the regenerators and the pumping system in order to avoid relative movements in the fluid circuit system. The bottom slider is a 42 step screw-motor with a maximum acceleration of $0.3 \mathrm{~g}$ and a maximum velocity of $300 \mathrm{~mm} \mathrm{~s}^{-1}$.

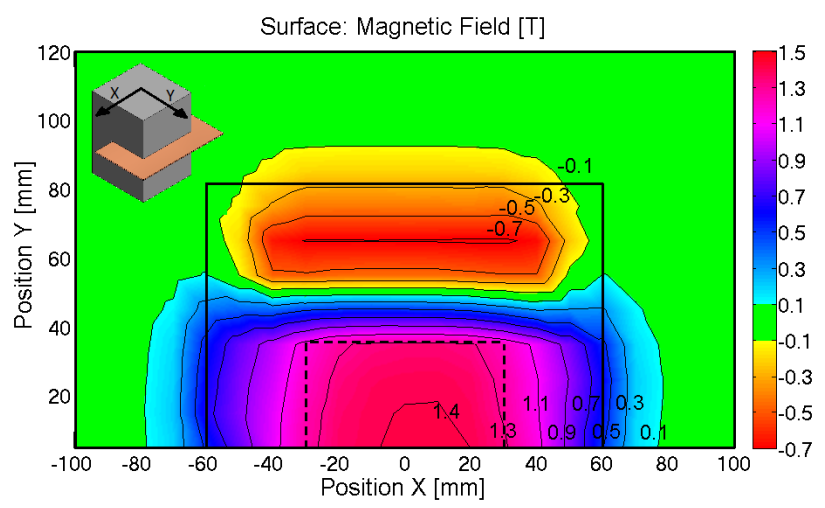

Figure 2: Magnetic field across a horizontal section of the magnet slot. The plane represented can be seen in the upper left corner of the figure. The solid line shows the edges of the slot. The dashed line represents the useful area of high field to place the regenerator

The intensity of the magnetic field has been measured with a transversal Hall probe, taking measurements in a grid of $(5 \times 5) \mathrm{mm}^{2}$ on the central plane of the slot. A map of the field is shown in Fig. 2. The magnet gives a high and uniform magnetic field of $(1.3-1.4)$ $\mathrm{T}$ in a surface of $(60 \times 35) \mathrm{mm}^{2}$. This is delimited by a dashed line in the figure and is the useful area to place the regenerator to be magnetised. Outside, the magnetic field falls rapidly and reaches zero field (between 0.1 and $-0.1 \mathrm{~T}$ ) $20 \mathrm{~mm}$ out of the edge of the magnet (green 
Table 1: Physical parameters of the double active magnetic regenerator

\begin{tabular}{ccccccc}
\hline Regenerator & Mass & $\begin{array}{c}\text { Interstitial } \\
\text { volume } \\
{\left[\mathrm{cm}^{3}\right]}\end{array}$ & $\begin{array}{c}\text { Housing } \\
\text { volume } \\
{\left[\mathrm{cm}^{3}\right]}\end{array}$ & $\begin{array}{c}\text { Porosity } \\
{[\%]}\end{array}$ & $\begin{array}{c}\text { Dead Volume } \\
(\text { hot } / \text { cold) } \\
{\left[\mathrm{cm}^{3}\right]}\end{array}$ & $\begin{array}{c}\text { Dead Volume Ratio } \\
\text { (hot / cold) } \\
{[\%]}\end{array}$ \\
\hline 1 & 15.22 & 1.26 & 3.19 & 39.6 & $(0.23 / 0.03)$ & $(18.2 / 2.4)$ \\
2 & 16.51 & 1.10 & 3.19 & 34.5 & $(0.23 / 0.06)$ & $(20.9 / 5.4)$
\end{tabular}

colour). So, the demagnetisation movement requires a displacement of $110 \mathrm{~mm}$ from the high to the low field region.

\subsection{Regenerator}

The chosen magnetocaloric material is gadolinium because it is a well-known benchmark material with a Curie temperature of $21^{\circ} \mathrm{C}$. Spheres of $0.2-0.4 \mathrm{~mm}$, obtained from Astronautics, were used. The physical parameters of the regenerators are summarised in Table 1. Gd masses of $15.22 \mathrm{~g}$ and $16.51 \mathrm{~g}$ were packed in the regenerator housings made of $\mathrm{PVC}$, having volumes of $3.19 \mathrm{~cm}^{3},(60 \times 10 \times 5.4) \mathrm{mm}^{3}$, and leaving interstitial volumes of 1.26 and $1.10 \mathrm{~cm}^{3}$. The resulting porosity of $39.6 \%$ and $34.5 \%$ are close to the theoretical $36 \%$ given by uniform spheres. The good compaction of the spheres prevents them from moving inside their housing under the action of the fluid and field. The thermal losses of the regenerator to the ambient are characterised by the relaxation time constants of around $150 \mathrm{~s}$ and $470 \mathrm{~s}$ in the positions inside and outside the magnet, respectively.

The dead volumes between the regenerator and the cold and hot sides have been carefully minimised because of the low interstitial volumes inside the regenerators. As described in section 2, the lower the dead volume in the hot and cold sides the better the system performance. It has also been explained that the pushed fluid volume ratio $\left(R_{V f}\right)$ should be higher than the dead volume ratio $\left(R_{V d}\right)$. Dead volume ratios around $20 \%$ and $4 \%$ have been achieved for the hot and cold sides, respectively.

\subsection{Fluid system}

The refrigerant liquid is $75 \%$ water $+25 \%$ ethylene glycol which is a common mixture as heat exchange liquid used with $\mathrm{Gd}$ because the corrosion is minimised. The pumping system is composed of two adjustable pistons to regulate their capacity to the range of the pushed fluid volume for the batch of experiments, and therefore avoid needless large volumes in the hot sides. Both pistons are pushed by an upper slider. Using pistons instead of a combination of pump and valves assures a precise regulation of the pushed volumes, which is critical in a device with such a small interstitial volume. The pistons have a diameter of $8 \mathrm{~mm}$ and a maximum stroke of $200 \mathrm{~mm}$, which allow pushing up to $10 \mathrm{~cm}^{3}$, more than enough for what is needed in these regenerators. The upper slider is a 42 step screw-motor with a maximum acceleration of $0.3 \mathrm{~g}$ and a maximum velocity of $600 \mathrm{~mm} \mathrm{~s}^{-1}$. The pressure drop of the regenerators is not a problem in the demonstrator due to the relatively low values of the cycle frequencies used (up to $0.82 \mathrm{~Hz}$ ).

\section{Experiments}

As it was seen before in Fig. 1(a) there are seven temperature probes (type E thermocouples) and one additional probe (Pt-100) to measure room temperature. Room temperature is controlled by an air conditioning system making sure it keeps between 26 and $28^{\circ} \mathrm{C}$ for all the experiments. The voltage signals are measured for each probe by a microvoltmeter using a scanner. A complete measurement of all the probes is taken in 2.8 seconds. Faster measurements can be taken if the number of channels to be read decreases. Experiments are automated and can be programmed in a batch, so the system is able to detect when a steady state (steady temperature span) has been reached or when an overheating in the cold side is produced, stopping the experiment and beginning the next one in the batch. Temperature logging, sliders control, heat load and automation of the system has been programmed in Labview.

Fig. 3 shows a typical experiment, where only channels $0-4$ are shown for clarity. Channels 5, 6 and 7 are similar to 3,2 and 1, respectively, but dephased half a cycle. The lines for the cold and hot side temperatures decrease and increase, respectively, until they reach a steady temperature span. The regenerator temperatures at $1 / 3$ and $2 / 3$ of its length are between both lines and represent the temperature of the magnetocaloric material. These are characterised by a high oscillation amplitude because of the temperature gradient of the liquid flowing through the regenerator. The oscillation frequency represented in the plot does not correspond to the real oscillation. They result from the different pe- 
riods of the data taking $(2.8 \mathrm{~s})$ and the operation cycle (2.86 s). In general, the experiments take up to $25 \mathrm{~min}$ utes to reach the steady state, but as they are launched in a batch, as seen later in section 5 , the regenerator temperature does not come back to room temperature and the next experiment does not start from zero temperature span. In fact, they take around 10 minutes. The steady state of the experiment is considered to be reached when the temperature span trend decreases below $0.05^{\circ} \mathrm{C} \mathrm{min}^{-1}$.

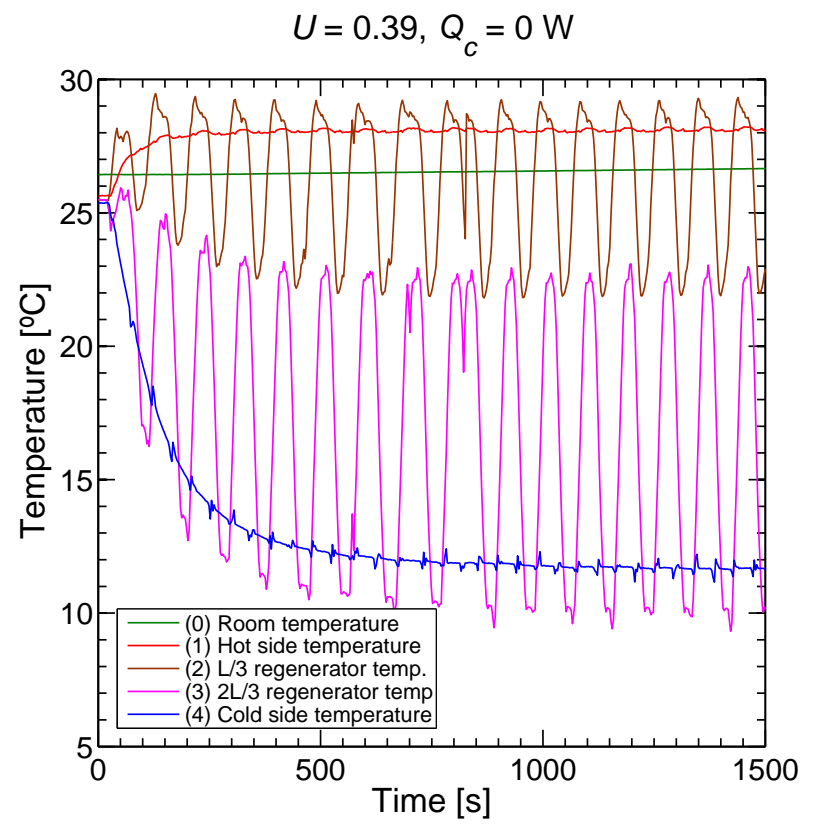

Figure 3: Typical temperature evolution of channels 0 to 4

Fast measurements can be taken if the scanner is fixed to measure only one channel. Up to 6.5 measurements per second can be taken by the microvoltmeter with acceptable accuracy. This allows studying each channel with high precision in order to analyse the effect of each particular action. Fig. 4 shows in detail channels 1, 2, 3 and 4 for a full cycle, where one can clearly see the steps of the cycle for regenerator 1 .

Fig. 4(a) shows the temperature of the hot side. It shows very slight temperature changes along the cycle.

In Figs. 4(b) and c) can be seen the temperature evolution inside of the regenerator at $\mathrm{L} / 3$ and $2 \mathrm{~L} / 3$. The magnetisation $\left(t_{1}\right)$ and demagnetisation $\left(t_{3}\right)$ steps show a sudden increase and decrease of the temperature of the regenerator of almost $1{ }^{\circ} \mathrm{C}$, respectively. Although, the temperature variation of the $\mathrm{Gd}$ under a magnetic field of $1.4 \mathrm{~T}$ is up to $4.7^{\circ} \mathrm{C}$ according to the summary figure

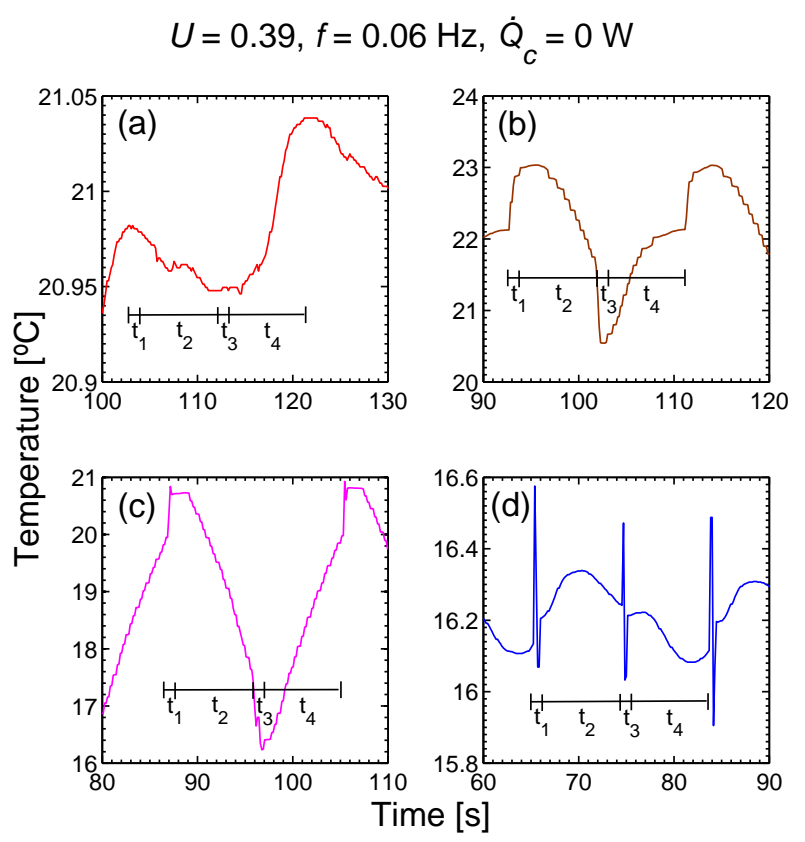

Figure 4: Details of temperature oscillations in a slow cycle: a) Hot side temperature of regenerator 1 (channel 1), b) temperature of regenerator 1 at L/3 (channel 2), c) temperature of regenerator 1 at $2 \mathrm{~L} / 3$ (channel 3) and d) cold side temperature (channel 4). The four steps of the cycle are defined as $t_{1}$ : Magnetisation, $t_{2}$ : Hot blow, $t_{3}$ : Demagnetisation and $t_{4}$ : Cold blow.

in Pecharsky and Gschneidner [13], one has to consider that the material is surrounded by a fluid with a high heat capacity. The hot blow $\left(t_{2}\right)$ shows a decrease of the temperature because of the heat release to the fluid, while the cold blow $\left(t_{4}\right)$ show an increase because of the heat absorption from the fluid. The amplitude of the temperature oscillation of the blow steps grows as the temperature span of the regenerator increases and until the steady state is reached.

The temperature evolution of the cold side can be seen in Fig. 4(d). As the cold side is placed between both regenerators, it receives the contribution of the cold blow from the regenerator $1\left(t_{4}\right)$ and from the regenerator 2 , which occurs in the hot blow of the regenerator 1 $\left(t_{2}\right)$, because it is dephased half a cycle. It shows a particular effect due to the small asymmetry in the regenerator masses and dead volumes of the cold side. The cold blow of the regenerator $1\left(t_{4}\right)$ achieves a colder temperature at the end of the step, while the cold blow of the regenerator $2\left(t_{2}\right)$ produces a temperature oscillation at slightly higher temperatures. As explained in section 3.2 both regenerators are not exactly equal, so, at the final steady state one of them is cooling down but the other one is not anymore, compensating each other. 
Table 2: Cycle frequencies $(\mathrm{Hz})$ for different mass flow rates and utilization factors $(U)$ or the corresponding strokes (mm)

\begin{tabular}{cc|cccccccc}
\hline $\begin{array}{c}\text { Mass flow } \\
\text { rate }\left[\mathrm{g} \mathrm{s}^{-1}\right]\end{array}$ & $\begin{array}{c}\text { Velocity } \\
{\left[\mathrm{mm} \mathrm{s}^{-1}\right]}\end{array}$ & $\begin{array}{c}U=0.1 \\
(5 \mathrm{~mm})\end{array}$ & $\begin{array}{c}U=0.19 \\
(10 \mathrm{~mm})\end{array}$ & $\begin{array}{c}U=0.29 \\
(15 \mathrm{~mm})\end{array}$ & $\begin{array}{c}U=0.39 \\
(20 \mathrm{~mm})\end{array}$ & $\begin{array}{c}U=0.49 \\
(25 \mathrm{~mm})\end{array}$ & $\begin{array}{c}U=0.68 \\
(35 \mathrm{~mm})\end{array}$ & $\begin{array}{c}U=0.88 \\
(45 \mathrm{~mm})\end{array}$ & $\begin{array}{c}U=1.1 \\
(55 \mathrm{~mm})\end{array}$ \\
\hline 0.26 & 5 & 0.38 & 0.22 & 0.15 & 0.12 & 0.09 & 0.07 & 0.05 & 0.04 \\
0.52 & 10 & 0.54 & 0.35 & 0.26 & 0.21 & 0.17 & 0.13 & 0.10 & 0.08 \\
1.04 & 20 & 0.68 & 0.51 & 0.41 & 0.34 & 0.29 & 0.23 & 0.18 & 0.16 \\
2.61 & 50 & 0.82 & 0.70 & 0.62 & 0.55 & 0.49 & 0.41 & 0.35 & 0.31
\end{tabular}

Pushed fluid volume ratio [Dimensionless]
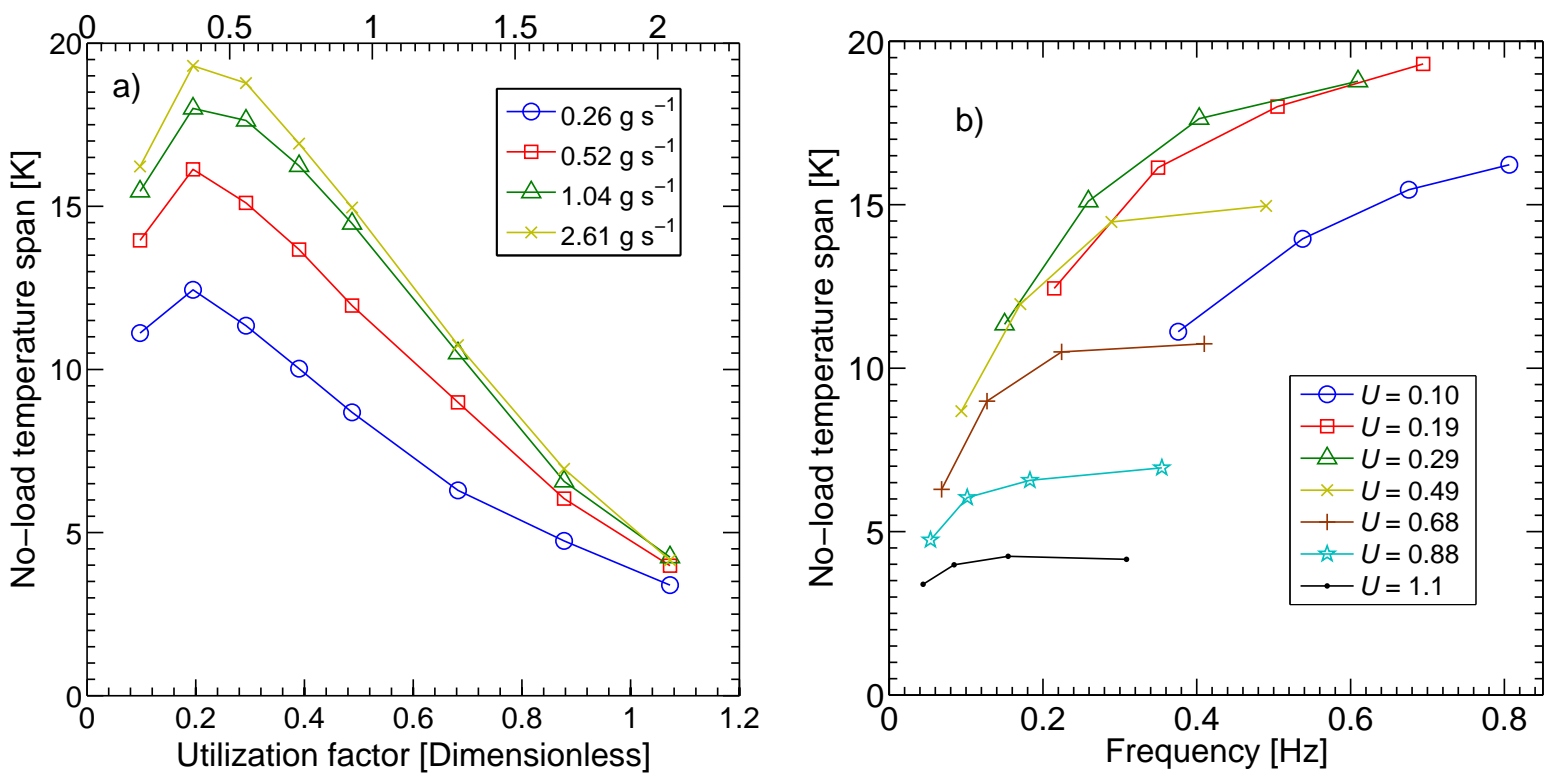

Figure 5: No-load temperature span as a function of a) utilization factor or pushed fluid volume ratio at several mass flow rates and b) frequency at several utilization factors.

Some spikes can be observed during magnetisation $\left(t_{1}\right)$ and demagnetisation $\left(t_{3}\right)$ in channel 4 (also in the peaks of channel 3 , and not so clearly in channel 2 ). They are due to the movement of the sensing thermocouples in and out of the magnetic field when the regenerator is magnetised and demagnetised, generating an e.m.f. in the thermocouples. Thermocouple 1 (and 7) remains out of the magnet all the time, so, it does not show such spikes.

\section{Results and discussion}

A series of experimental results have been done programming a batch of experiments, changing the following parameters:

a) Utilization factor: $(0.1,0.19,0.29,0.39,0.49,0.68$, $0.88,1.1)$

b) Mass flow rate: $(0.26,0.52,1.04,2.61) \mathrm{g} \mathrm{s}^{-1}$ c) Heat load: $(0,0.5,1,1.5,2,2.5,3,3.5,4,5,6,7,8)$ $\mathrm{W}$

The operation frequencies of the demonstrator for different utilization factors and mass flow rates can be seen in Table 2. The lower limit for the magnetisation / demagnetisation time is $0.48 \mathrm{~s}$ due to the characteristics of the bottom slider described in section 3.1. The fluid blow periods can be obtained from the frequency values of the table. The table also shows other equivalent parameters like the piston velocities, proportional to the mass flow rates, and the strokes that correspond to the different $U$ values. Taking into account the combinations of these two parameters and the heat load, a total of 256 experiments have been done. Some interesting plots will be discussed below to obtain conclusions about the influence of each parameter and their optimisation regarding temperature span, cooling power, $C O P$ and thermodynamic cycles. 

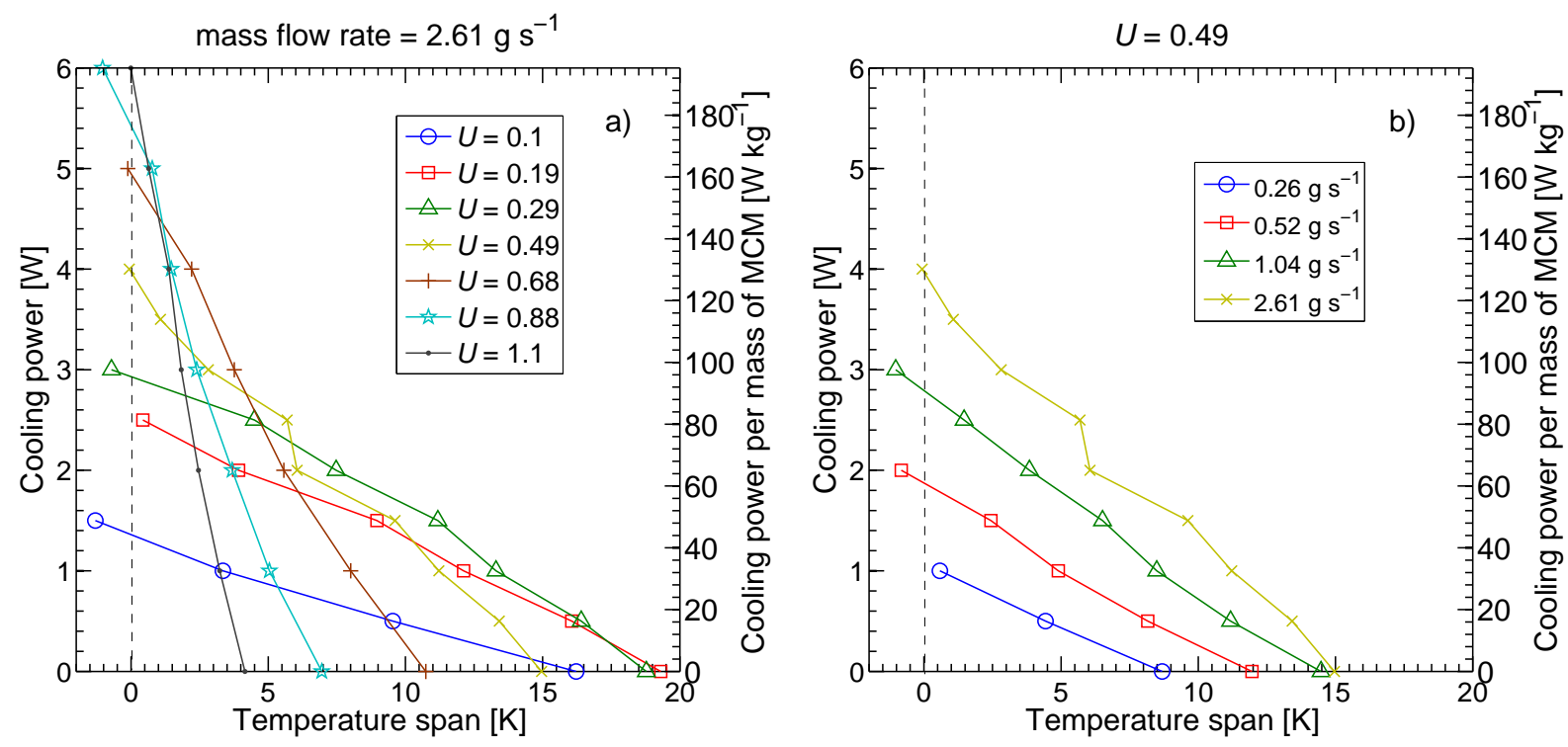

Figure 6: Cooling power - Temperature span relationship as a function of a) utilization factor at $2.61 \mathrm{~g} \mathrm{~s}^{-1}$ and b) mass flow rate at $U=0.49$. Points to the left of the dashed lines mean that the hot side temperature is lower than the cold side temperature

\subsection{No-load temperature span}

The temperature spans obtained in the experiments with no heat load for the various utilization factors and fluid mass flow rates have been represented as a function of $U$ in Fig. 5(a) and as a function of frequency in Fig. 5(b).

Fig. 5(a) shows that a maximum no-load temperature span of $19.3 \mathrm{~K}$ has been achieved, which means a regeneration ratio of $\xi=4.1$. The optimum utilization factor for each mass flow rate is $U=0.19$. Higher values decrease gradually the temperature span because the regenerator capacity is progressively overwhelmed. It agrees with some publications showing that the maximum no-load temperature span is obtained at low utilization factors (Bahl et al. [14], Nielsen et al. [15]). Fig. 5(a) can also be seen as function of the pushed fluid volume ratio. Taken the most adverse dead volume ratio around 0.2 , which is the average value from the hot sides (see Table 1), one can see that the no-load best results are obtained between $R_{V d}(0.2)$ and $1+R_{V d}$ (1.2) as was suggested in section 2. Higher values than $1+R_{V d}$ make the fluid from the hot side to reach the cold side and vice versa, reducing substantially the temperature span. Lower values than $R_{V d}$ make that the fluid from the regenerator does not reach the hot sides.

The curves represented in Fig. 5(a) show a gradual improvement of the temperature span with the mass flow rate or the frequency. This span improvement is more clearly seen in Fig. 5(b) for every utilization factor in the frequency range reached with our demonstrator. This happens while the heat transfer deterioration between magnetocaloric material and refrigerant due to frequency increase is compensated by the heat loss reduction to the ambient on each cycle. For higher frequencies the behaviour should worsen, but unluckily we were not able to see it because our demonstrator cannot reach the higher frequencies required. The good heat transfer existing for the small spheres of our regenerator maintains the span increasing up to high frequencies. Fig. 5(b) shows a tendency to reach the optimum temperature span at lower operation frequencies for increasing utilization factors. The maximum of the span is reached only in the case $U=1.1$ and the other curves show a diminishing slope on increasing $U$.

\subsection{Cooling power}

A maximum cooling power of $6 \mathrm{~W}$ at zero temperature span has been achieved, which results in $189 \mathrm{~W}$ per $\mathrm{kg}$ of magnetocaloric material. The correlation of the temperature span with the cooling power can be observed in Fig. 6. It is clear, as is shown for regenerators with one magnetocaloric material in Nielsen et al. [16], that exists a lineal correlation between $\Delta T$ and $\dot{Q}_{c}$. Fig. 6(a) also shows that the slope of the lines increases as the utilization factor is increased, which means that higher cooling powers can be achieved, but lower tem- 

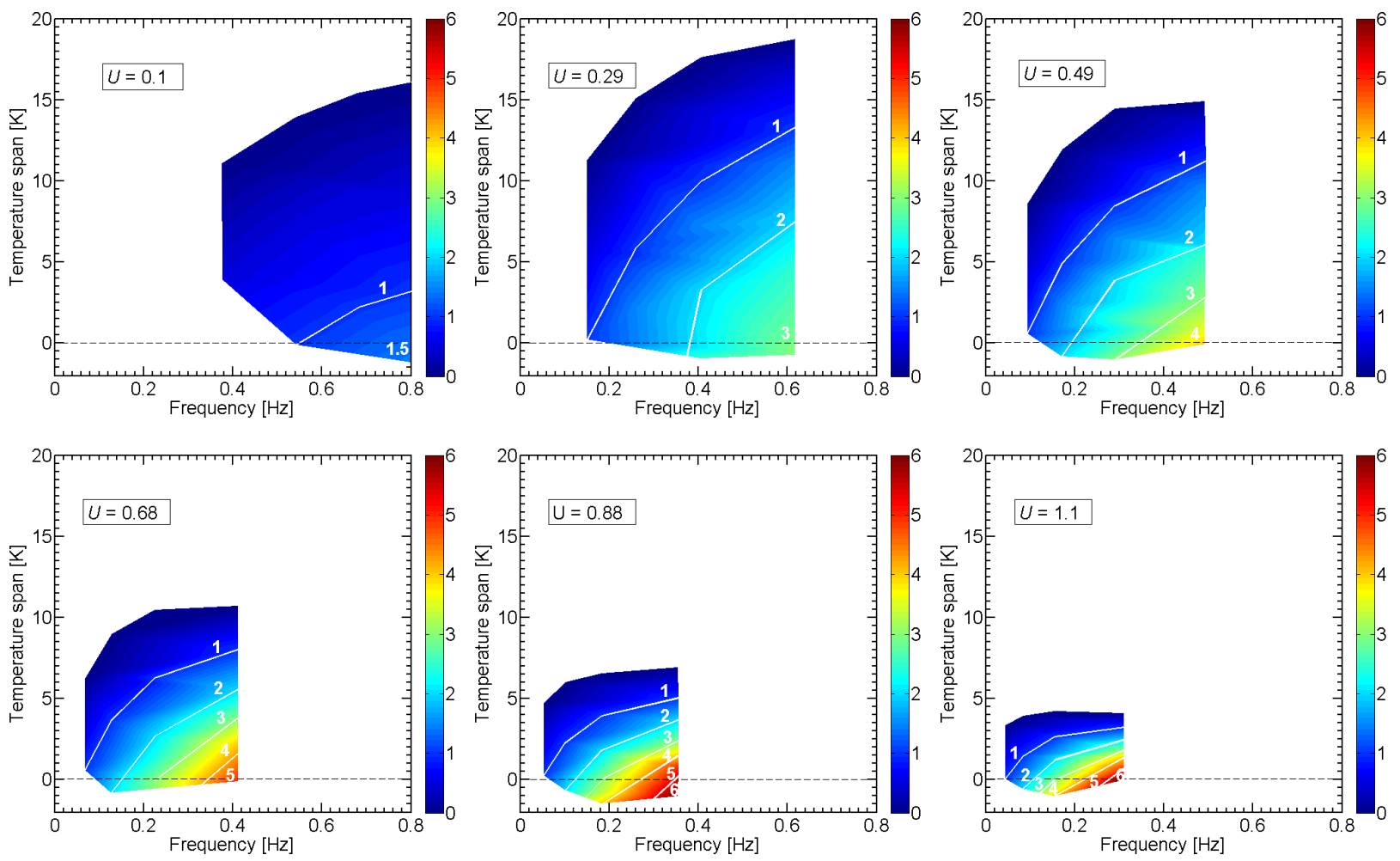

Figure 7: Cooling power surface as a function of temperature span and frequency for several utilization factors. Level lines represent equal values of cooling power.

perature spans are reached at the same time. Summarising, a high utilization factor is desirable when demanding a high cooling power, as long as the achieved temperature span is enough, and a low utilization factor is desirable when demanding a high temperature span, as long as the achieved cooling power is enough.

On the other hand, in Fig. 6(b) one can observe that the temperature span and the cooling power improve as the mass flow rate is increased. Increasing the mass flow rate at constant utilization factor increases the frequency (as can be seen in Table 2), leading to the cooling power improvement. Also the slope of the curves increases with the mass flow rate. For higher mass flow rates than the values used, some intersections of the lines would appear as happens in Fig. 6(a), showing some optimum ranges of the mass flow rate for given values of the temperature span. This cannot be seen because of the frequency limitation of the demonstrator.

In order to see the effect of the frequency on the correlation of the cooling power with the temperature span, surfaces of cooling power for several utilization factors have been included in Fig. 7. It is clear that increasing the frequency within our frequency range, the cool- ing power increases for every utilization factor, as the cold side is able to absorb a higher heating power. Also, they show that wider temperature spans are achieved for higher frequencies as was shown in Fig. 5 for the noload case. Moreover, higher cooling powers can be obtained for higher utilization factors, even though lower frequencies are reached because of the frequency limitations. In fact, the cooling power would be higher if the operation frequency could be increased.

\subsection{Coefficient of performance (COP)}

It was not the aim of this demonstrator to optimise the $C O P$, because the motors were selected to be as versatile as possible in order to change the operation parameters easily. In any case, the calculation of the $C O P$ of the demonstrator has been included to give a comprehensive parameter study. The electrical work is obtained by measuring the intensity consumption of the motors. Fig. 8 shows the maximum $C O P$ and the corresponding cooling power as a function of frequency, indicating the optimum utilization factors at several demanded temperature spans. It represents a real application chart where one can determine the necessary operation fre- 
quency of the machine to obtain the required cooling power for a certain demanded temperature span. The experimental temperature spans have been interpolated to obtain this figure. A maximum $C O P$ of $0.7\left(\dot{Q}_{c}=\right.$ $6 \mathrm{~W}, \Delta T=0 \mathrm{~K}$ ) was obtained at $U=1.1, f=0.3$ $\mathrm{Hz}$. As was show in section 5.2, the cooling power increases with the frequency. The $C O P$ has a similar behaviour. The cooling power and $C O P$ curves do not show maxima in the figure corresponding to optimised frequencies because of the low frequency limitation. It is expected that, in a hypothetical experiment without frequency limitations, optimum cooling power and $C O P$ values would be obtained for some higher frequencies. According to Kitanovski et al. [17], the optimal frequency for the cooling power is higher than for the $C O P$ values, and this is expected from the higher slope of the cooling power curves compared to those of $C O P$ in the high frequency range of Fig. 8. One can also observe that on increasing the required temperature span the cooling power capacity decreases considerably and its dependence with frequency turns weaker, at the same time that the optimum utilization factor decreases also.

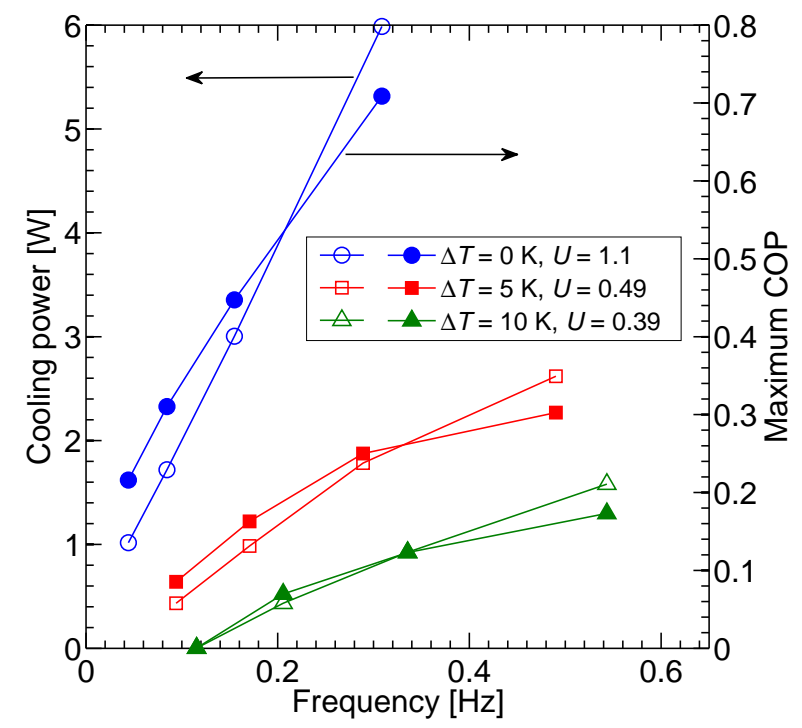

Figure 8: Maximum $C O P$ and the corresponding cooling power as a function of frequency for several temperature spans.

\subsection{Thermodynamic cycles}

Several thermodynamic cycles have been studied experimentally in order to check the behaviour of the system modifying the typical Brayton-AMR cycle, as was suggested by Kitanovski et al. [18] in a simulation study.
All the experiments analysed above have been done following a Brayton-AMR cycle, which is explained in section 3 and can be seen in the thermodynamic scheme represented in Fig. 9(a). It is an entropy - temperature plot, where magnetisation (from $\mathrm{M}_{\mathrm{i}}$ to $\mathrm{M}_{\mathrm{f}}$ ) and demagnetisation (from $D_{i}$ to $D_{f}$ ) steps are represented as adiabatic temperature increase and decrease, respectively. Hot blow (from $\mathrm{HB}_{\mathrm{i}}$ to $\mathrm{HB}_{\mathrm{f}}$ ) and cold blow $\left(\mathrm{CB}_{\mathrm{i}}\right.$ to $\mathrm{CB}_{\mathrm{f}}$ ) are isofield steps with a decrease and increase of temperature, respectively. In this cycle, each step starts when the previous one have finished, as can also be seen in the sequence diagram of the Fig. 9(e). Once in the magnetisation / demagnetisation steps the magnetic fields $H_{\max }$ and $H_{0}$ have been reached, respectively, the blow steps are activated. Similarly, when the fluid blows have finished (zero mass flow rate), the magnetisation / demagnetisation steps are activated.

Three additional type of cycles, based on the Brayton-AMR one, have been realised introducing various phase differences between the magnet and the fluid flow steps. The group of experiments have been done for the fixed parameters $U=0.49$ and $\dot{m}=1.04 \mathrm{~g} \mathrm{~s}^{-1}$, but modifying the thermodynamic cycle as seen in Fig. 9(b), (c), and (d). The figures are out of scale and show schematically the thermodynamic steps of the different cycles. These new cycles are obtained by programming the position of the upper slider moving the pistons (PF), and the bottom slider moving the regenerator in and out of the magnet (PM). When the slider is at a given percentage from the end of its movement the next action is launched before the completion of the previous step. Therefore, different and faster cycles are obtained.

Fig. 9(b) is characterised by the control of the relative position of the magnet (PM), which means that when the magnetisation and demagnetisation movements are at a given percentage from their final position, the next step is activated, that is to say the hot and cold blows, respectively. So, the fluid blows are initiated before the magnetisation or demagnetisation processes had ended. Thus, the cycle is faster and is modified as shown in Fig. 9(b). A second type of cycle, shown in Fig. 9(c) is characterised by controlling the position of the pistons giving the fluid blows (PF). When the pistons movement are at a given percentage from the end of their strokes, the next step is activated, magnetisation or demagnetisation. So, the magnetic field activation is initiated before the fluid flow has ended, modifying the cycle as given in Fig. 9(c) and reducing the cycle time again.

Finally, a third type of cycle, shown in Fig. 9(d) is a combination of the two previous cycles by controlling the relative position of the magnet and the position of the pistons starting the following steps before the end of 

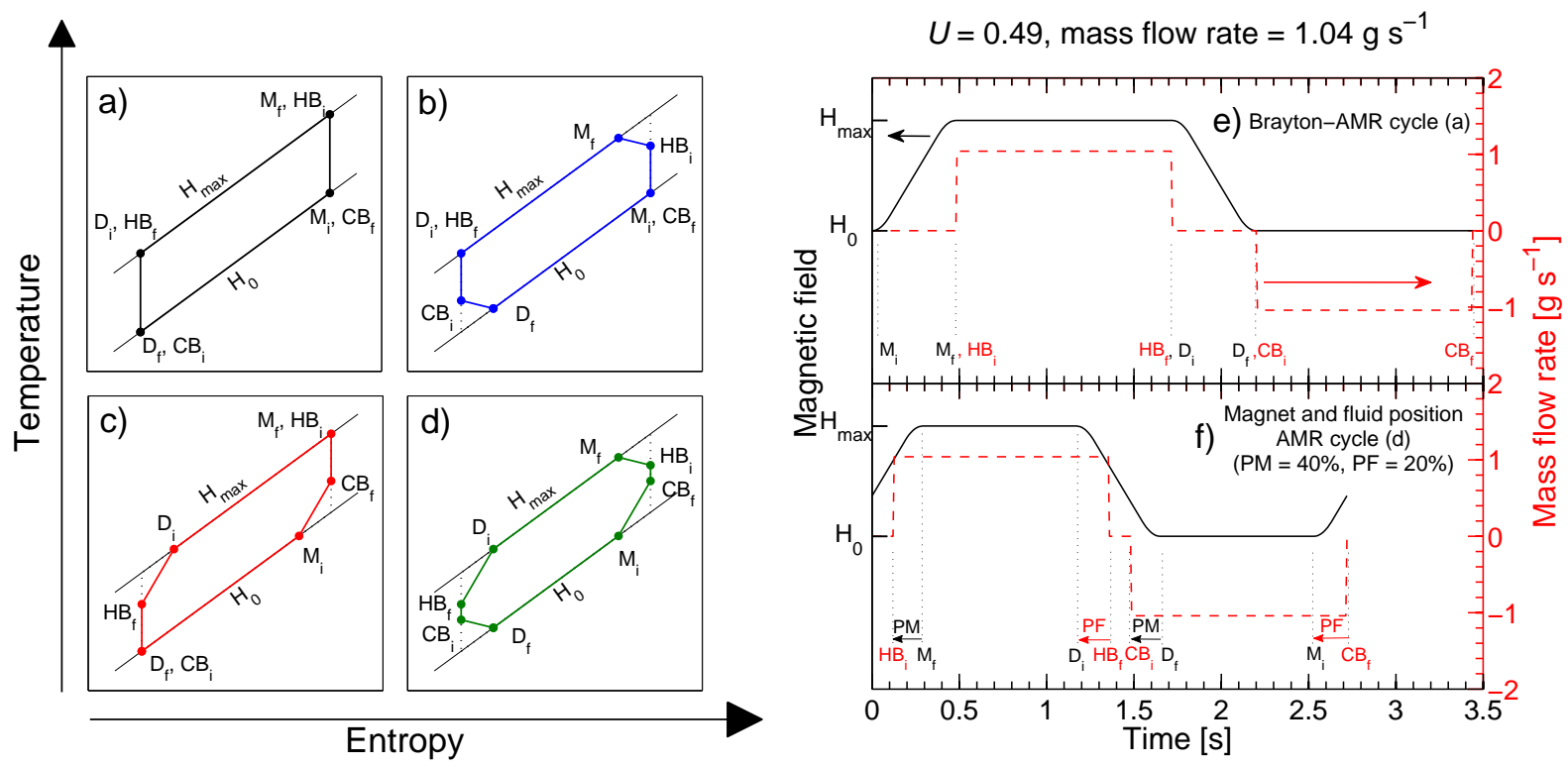

Figure 9: Thermodynamic schemes of the cycles: a) Brayton-AMR cycle, b) magnet position AMR cycle, c) fluid position AMR cycle and d) magnet and fluid position AMR cycle. The meaning of the symbols are M: magnetisation, D: demagnetisation, CB: cold blow, and HB: hot blow, which are combined with subscripts to denote initial (i) and final (f) process. The experimental sequence diagrams over one complete cycle referenced to the a) and d) cycles can be seen in e) and f) $\left(U=0.49\right.$ and $\left.\dot{m}=1.04 \mathrm{~g} \mathrm{~s}^{-1}\right)$. PM: refers to programming the relative position of the magnet (fluid blow starts at a given percentage from the final position of the relative magnet movement), and PF: refers to programming the position of the pistons giving the fluid blows (relative magnet movement starts at a given percentage from the final position of the pistons movement).

their movements (PM and PF, respectively). The cycle time of this option is the fastest one. Fig. 9(f) shows the sequence diagram of the latter one for the case of $\mathrm{PM}=40 \%$ and $\mathrm{PF}=20 \%$. One can see how the programming of PM and PF modify the original one (Fig. 9(e)) starting the blow steps before the magnetisation / demagnetisation steps have ended (PM) and vice versa (PF). As a result, the cycle time is reduced as can be seen comparing the latter two figures.

All these modifications reduce the area of the cycles and, consequently, the input work. The cooling results of the modified cycles compared with the BraytonAMR cycle can be seen in Fig. 10. The cycle time for each option is included in the legend. They show consistent trends to improve the cooling power and temperature span. Similar results are shown by Plaznik et al. [7] in some cycles of a simulation and experimental study. Considering the results given in the figure, one can see that the slope of the lines representing the cooling power versus the temperature span do not change significantly with the different cycles. Only a small change is observed for low cooling powers. The cycle represented in Fig. 9(b) clearly improves the results by starting the fluid blow at $20 \%$ of the final position of the field, and even better starting at $40 \%$. The cycle given in Fig. 9(c) gives only small improvements for the activation of the field before the given $10 \%$ and $20 \%$ of the end of the fluid blows. Finally, intermediate results are obtained for the cycle shown in Fig. 9(d). The frequency increase of the modified cycles, being up to $27 \%$ faster for the $\mathrm{PM}=40 \%$ and $\mathrm{PF}=20 \%$ case, is partly responsible for the improved performance of the modified cycles.

As a result of this partial study of modified cycles one can see the potential of introducing modifications to improve the cycle behaviour. A systematic and deeper study should be done to check the influence of different modifications of the cycle in the results for the cooling power, temperature span, and COP. An effort should be done to run the different cycles with the same operation frequency in order to compare them more reliably. The blow and / or magnetisation - demagnetisation steps should be lengthen to compensate for the overlap of the cycle steps. It would also be interesting to carry out isothermal steps to compare with other standard AMR cycles as Carnot, Ericsson or a mixture of them. 


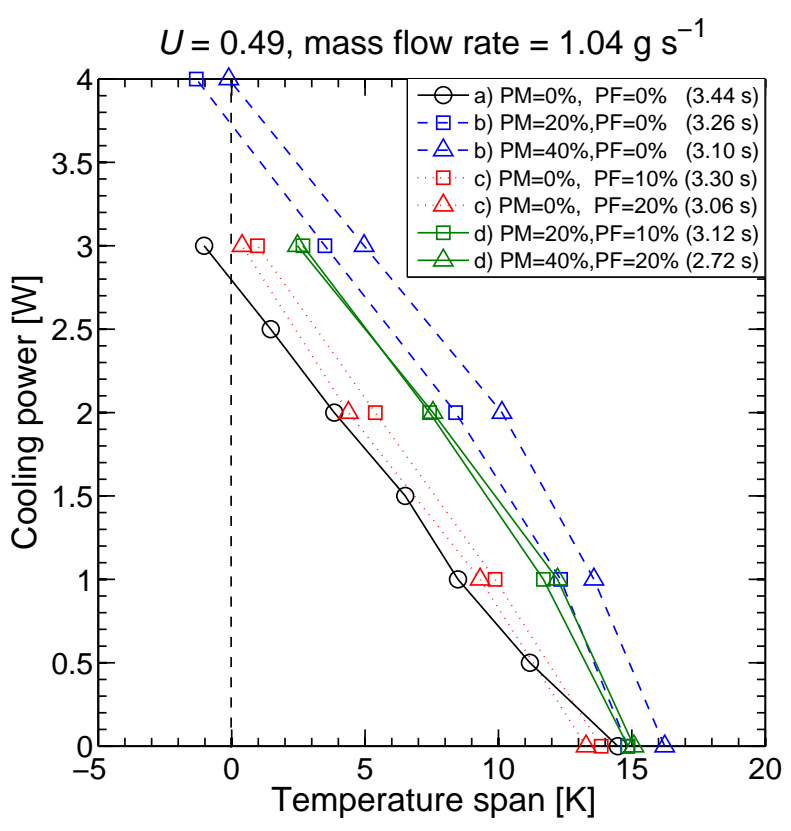

Figure 10: Cooling power - temperature span behaviour of different thermodynamic cycles a) to d) for the fixed operation parameters $U=$ 0.49 and $\dot{m}=1.04 \mathrm{~g} \mathrm{~s}^{-1}$. Lettering of the legend refers to the cycles showed in Fig. 9. Cycle time for each option is also included between brackets in the legend.

\section{Conclusion}

In this paper, a new versatile magnetic refrigeration demonstrator is presented and a comprehensive study of its performance with $30 \mathrm{~g}$ of Gadolinium as magnetocaloric material. It is a first generation system based on a double active magnetic regenerator with a permanent magnet of $1.4 \mathrm{~T}$. It achieved a maximum no-load temperature span of $19.3 \mathrm{~K}(\xi=4.1)$ at $U=0.19$ and $\dot{m}=2.61 \mathrm{~g} \mathrm{~s}^{-1}$ and a maximum cooling power at zero temperature span of $6 \mathrm{~W}\left(189 \mathrm{~W} \mathrm{~kg}^{-1}\right)$ at $U=1.1$ and $\dot{m}=2.61 \mathrm{~g} \mathrm{~s}^{-1}$. Also COP values have been presented with an optimum value of $0.7\left(\dot{Q}_{c}=6 \mathrm{~W}, \Delta T=0 \mathrm{~K}\right)$ at $U=1.1$ and $\dot{m}=2.61 \mathrm{~g} \mathrm{~s}^{-1}$ although its optimisation was not the aim of the demonstrator. Besides, different modified Brayton-AMR cycles have been tested, showing the potential to improve the magnetic refrigeration behaviour. The temperature span and cooling power capacity are remarkable compared to first generation systems of the literature. Accurate temperature measurements of sinks and magnetocaloric material can be recorded allowing a detailed analysis of the heat transfer and the dynamics of the system. The low frequencies achieved by the demonstrator, as a reciprocating system, are the main limitation to obtain better results.
This work helps advancing in future scalable magnetic refrigeration prototypes regarding $C O P$, cooling power and temperature span. A magnet with a wider space to introduce more magnetocaloric material and more advanced mechanics to reach higher frequencies seems to be critical for the design of a useful prototype. Also, the choice of the proper AMR cycle has to be taken into account to maximise the performance of the system. Other options as Tomc et al. [19] presents to solve problems of fast heat transfer between solid and liquid through thermal switch technology should be taken into account.

\section{Acknowledgements}

We are grateful to BSH Electrodomesticos España S.A. (Contract 2013/0011), MEC (project MAT201344063-R) and DGA (consolidated group E100) for financial support. Astronautics Technology Center is also acknowledged for providing the Gadolinium spheres used in the demonstrator.

\section{References}

[1] K. Gschneidner, V. Pecharsky, Thirty years of near room temperature magnetic cooling: Where we are today and future prospects, Int. J. Refrigeration 31 (2008) 945-961.

[2] B. Yu, M. Liu, P. Egolf, A. Kitanovski, A review of magnetic refrigerator and heat pump prototypes built before the year 2010 , Int. J. Refrigeration 33 (2010) 1029-1060.

[3] F. Scarpa, G. Tagliafico, L. Tagliafico, Classification proposal for room temperature magnetic refrigerators, Int. J. Refrigeration 35 (2012) 453-458.

[4] D. W. Lu, X. N. Xu, H. B. Wu, X. Jin, A permanent magnet magneto-refrigerator study on using Gd / Gd-Si-Ge / Gd-Si-GeGa allows, in: Proceedings of the First IIF-IIR International Conference on Magnetic Refrigeration at Room Temperature (2005) , Montreux, Switzerland, pp. 291-296.

[5] S. Bour, J. Hamm, H. Michot, C. Muller, Experimental and numerical analysis of a reciprocating room temperature active magnetic regenerator, in: Proceedings of the Third IIF-IIR International Conference on Magnetic Refrigeration at Room Temperature (2009), Des Moines, IO, U.S.A., pp. 415-424.

[6] Y. Chiba, A. Smaili, C. Mahmed, M. Balli, O. Sari, Thermal investigations of an experimental active magnetic regenerative refrigerator operating near room temperature, Int. J. Refrigeration 37 (2014) 36-42.

[7] U. Plaznik, J. Tušek, A. Kitanovski, A. Poredoš, Numerical and experimental analyses of different magnetic thermodynamic cycles with an active magnetic regenerator, Appl. Therm. Eng. 59 (2013) 52-59.

[8] J. Tušek, A. Kitanovski, S. Zupan, I. Prebil, A. Poredoš, A comprehensive experimental analysis of gadolinium active magnetic regenerators, Appl. Therm. Eng. 53 (2013) 57-66.

[9] R. Bjørk, C. Bahl, A. Smith, N. Prys, Optimization and improvement of Halbach cylinder design, J. Appl. Phys. 104 (2008) 13910.

[10] A. Tura, A. Rowe, Permanent magnet magnetic refrigerator desgin and experimental characterization, Int. J. Refrigeration 34 (2011) 628-639. 
[11] M. Richard, A. Rowe, R. Chahine, Magnetic refrigeration: Single and multimaterial active magnetic regenerator experiments, J. Appl. Phys. 4 (2004) 2146-2150.

[12] A. Tishin, Y. Spichkin, The magnetocaloric effect and its applications, Institute of Physics Publishing, Bristol and Philadelphia (2003).

[13] V. Pecharsky, K. Gschneidner, Adavanced magnetocaloric materials: What does the future hold?, Int. J. Refrigeration 29 (2006) 1239-1249.

[14] C. Bahl, T. F. Petersen, N. Pryds, A. Smith, A versatile magnetic refrigeration test device, Rev. Sci. Instrum. 79 (2008) 093906.

[15] K. Nielsen, C. Bahl, A. Smith, R.Bjørk, N. Pryds, J. Hattel, Detailed numerical modeling of a linear parallel-plate active magnetic regenerator, Int. J. Refrigeration 32 (2009) 1478-1486.

[16] K. Nielsen, C. Bahl, A. Smith, N. Pryds, J. Hattel, A comprehensive parameter study of an active magnetic regenerator using a 2d numerical model, Int. J. Refrigeration 33 (2010) 753-764.

[17] A. Kitanovski, J. Tušek, U. Tomc, U. Plaznik, M. Ožbolt, A. Poredoš, Magnetocaloric energy conversion: From theory to applications, Springer International Publishing (2015).

[18] A. Kitanovski, U. Plaznik, J. Tušek, A. Poredoš, New thermodynamic cycles for magnetic refrigeration, Int. J. Refrigeration 37 (2014) 28-35.

[19] U. Tomc, J. Tušek, A. Kitanovski, A. Poredoš, A new magnetocaloric refrigeration principle with solid-state thermoelectric thermal diodes, Appl. Therm. Eng. 58 (2013) 1-21. 